\title{
Development of a Full-Thickness Human Gingiva Equivalent Constructed from Immortalized Keratinocytes and Fibroblasts
}

\author{
Jeroen K. Buskermolen, MSc, ${ }^{1,2}$ Christianne M.A. Reijnders, PhD, ${ }^{3}$ Sander W. Spiekstra, BSc, \\ Thorsten Steinberg, PhD, Cornelis J. Kleverlaan, PhD, Albert J. Feilzer, PhD, DMD, \\ Astrid D. Bakker, $\mathrm{PhD}$, and Susan Gibbs, $\mathrm{PhD}^{1,3}$
}

Organotypic models make it possible to investigate the unique properties of oral mucosa in vitro. For gingiva, the use of human primary keratinocytes $(\mathrm{KC})$ and fibroblasts (Fib) is limited due to the availability and size of donor biopsies. The use of physiologically relevant immortalized cell lines would solve these problems. The aim of this study was to develop fully differentiated human gingiva equivalents (GE) constructed entirely from cell lines, to compare them with the primary cell counterpart (Prim), and to test relevance in an in vitro wound healing assay. Reconstructed gingiva epithelium on a gingiva fibroblast-populated collagen hydrogel was constructed from cell lines (keratinocytes: TERT or HPV immortalized; fibroblasts: TERT immortalized) and compared to GE-Prim and native gingiva. GE were characterized by immunohistochemical staining for proliferation (Ki67), epithelial differentiation (K10, K13), and basement membrane (collagen type IV and laminin 5). To test functionality of GE-TERT, full-thickness wounds were introduced. Reepithelialization, fibroblast repopulation of hydrogel, metabolic activity (MTT assay), and (pro-)inflammatory cytokine release (enzyme-linked immunosorbent assay) were assessed during wound closure over 7 days. Significant differences in basal KC cytokine secretion (IL-1 $\alpha$, IL-18, and CXCL8) were only observed between KC-Prim and KCHPV. When Fib-Prim and Fib-TERT were stimulated with TNF- $\alpha$, no differences were observed regarding cytokine secretion (IL-6, CXCL8, and CCL2). GE-TERT histology, keratin, and basement membrane protein expression very closely represented native gingiva and GE-Prim. In contrast, the epithelium of GE made with HPV-immortalized KC was disorganized, showing suprabasal proliferating cells, limited keratinocyte differentiation, and the absence of basement membrane proteins. When a wound was introduced into the more physiologically relevant GE-TERT model, an immediate inflammatory response (IL-6, CCL2, and CXCL8) was observed followed by complete reepithelialization. Seven days after wounding, tissue integrity, metabolic activity, and cytokine levels had returned to the prewounded state. In conclusion, immortalized human gingiva $\mathrm{KC}$ and fibroblasts can be used to make physiologically relevant GE, which resemble either the healthy gingiva or a neoplastic disease model. These organotypic models will provide valuable tools to investigate oral mucosa biology and can also be used as an animal alternative for drug targeting, vaccination studies, microbial biofilm studies, and testing new therapeutics.

\section{Introduction}

$\mathbf{T}$ HE ORAL MUCOSA forms the protective barrier of the oral cavity against harmful environmental influences (e.g., pathogens, chemicals, constant abrasion). ${ }^{1}$ To study the barrier properties of human oral mucosa, in vitro 3D organotypic cultures resembling the native tissue can be constructed from keratinocytes and fibroblasts isolated from biopsies. $^{2-7}$ However, the availability of primary human oral tissue for research is very limited, biopsies are small, and,

Departments of ${ }^{1}$ Oral Cell Biology, ${ }^{2}$ Dental Materials Science, Academic Centre for Dentistry Amsterdam (ACTA), University of Amsterdam and Vrije Universiteit Amsterdam, MOVE Research Institute Amsterdam, Amsterdam, The Netherlands.

${ }^{3}$ Department of Dermatology, VU University Medical Centre, Amsterdam, The Netherlands.

${ }^{4}$ Department of Oral Biotechnology, University Medical Center Freiburg, Freiburg, Germany.

Part of the data of this manuscript was used for an abstract entitled "Gingiva Equivalents Derived from Human TERT-Immortalized Keratinocytes and Fibroblasts for Investigation of Oral Wound Healing" for the 2015 TERMIS World Congress, which was published in Tissue Engineering Part A. September 2015.

(c) Jeroen K. Buskermolen et al. 2016; Published by Mary Ann Liebert, Inc. This Open Access article is distributed under the terms of the Creative Commons Attribution Noncommercial License (http://creativecommons.org/licenses/by-nc/4.0/) which permits any noncommercial use, distribution, and reproduction in any medium, provided the original author(s) and the source are credited. 
due to the origin of the material, it is often infected. Furthermore, once isolated, the primary cells enter senescence after only a small number of passages. The use of physiologically relevant immortalized cell lines, which maintain the properties of their primary cell counterpart, would overcome these problems. This would make larger scale experiments possible with organotypic oral mucosa models. Furthermore, the use of human cell line models complies with EU regulations, which encourage replacement, reduction, and refinement of animal models (EU Directive 2010/63/EU). Therefore, organotypic 3D models constructed from cell lines are required to investigate oral mucosa biology and can also be used as an animal alternative for drug targeting, vaccination studies, microbial biofilm studies, and testing new therapeutics. In this study, we developed a full-thickness oral gingiva equivalent (GE) constructed entirely from immortalized cell lines, which closely resembles the histology of the native tissue, to test its ability to close a full-thickness wound in vitro.

When developing a GE, it is important to take the characteristics of the native tissue into account. The stratified squamous epithelium of the gingiva is mainly composed of $\mathrm{KC}$ and is responsible for creating and maintaining a barrier with the outside environment. ${ }^{1,8}$ The different stages in terminal differentiation of $\mathrm{KC}$ in a $\mathrm{GE}$ from the basal layer to the outermost layers can be visualized by differential keratin expression. Keratins are important structural proteins, which not only give mechanical stability but are also involved in cell signaling, transport, and differentiation of keratinocytes. $^{8,9}$ The expression of keratin 10 and 13 (K10 and K13) indicates the presence of differentiated mucosal $\mathrm{KC}$ and the formation of a functional barrier. ${ }^{3,9}$ The epithelium is supported by the lamina propria, connective tissue that consists predominantly of extracellular matrix (ECM). The epithelium and lamina propria connect through the basement membrane. Important proteins within the basement membrane are laminin 5 and collagen type IV. ${ }^{10}$ Fibroblasts are located in the lamina propria where they maintain the ECM and interact, through soluble mediators, with $\mathrm{KC}$ and immune cells to coordinate tissue repair and immune reactions. ${ }^{11,12}$ Upon wounding, an innate immune response is triggered to initiate wound closure and mediate the infiltration of immune cells to counteract infection. As in skin, (pro-) inflammatory cytokines (e.g., TNF- $\alpha$, IL- $1 \alpha$, and IL-6) and chemokines (e.g., CXCL8, CCL2, CCL5, and CCL20) are secreted upon tissue injury. ${ }^{4} \mathrm{KC}$ proliferate and migrate from the wound edge, differentiate, and ultimately reepithelialize the wound. ${ }^{12}$ Fibroblast migration and tissue remodeling follow to restore tissue integrity. In this study, we aimed to reproduce this complex tissue architecture of native gingiva in a GE model.

Previously, we have described skin and gingiva models consisting of a reconstructed epithelium on a fibroblastpopulated connective tissue matrix constructed from primary cells, and recently we have described a skin equivalent (SE) model constructed from TERT-immortalized skin keratinocyte and fibroblast cell lines. ${ }^{3,5,13,14}$ The importance of using tissue-specific cells when making tissue equivalents is emphasized in our recent study describing different histology and cytokine release from SE and GE. ${ }^{4}$ This is further illustrated by the extensive review of Sriram et al., which discusses the heterogeneity of fibroblasts and the unique phenotype of oral mucosal fibroblasts and their potential role in wound healing. ${ }^{15}$ Therefore, to create functional GE (immortalized), tissue-specific cells must be used. To our knowledge, there is only one report combining immortalized human oral $\mathrm{KC}$ with immortalized human oral fibroblasts for the establishment of an organotypic model. ${ }^{16}$ These GE were made with HPV-immortalized cells, which lacked a well-defined differentiated epithelium. This has previously been reported to be a preneoplastic characteristic of HPVimmortalized cells. ${ }^{17-20}$ Immortalized human oral $\mathrm{KC}$ and fibroblasts have been used for in vitro studies on biocompatibility, cancer, and microbiome interaction. ${ }^{18,21,22}$ Both HPV and TERT-immortalized human oral KC have been described. With regards to TERT-immortalized oral keratinocytes, mainly the OKF6 cell line originating from the floor of the mouth has been used. ${ }^{23}$ The human gingiva cell line used in our study, OKG4/bmi1/TERT (further referred to as KC-TERT), was immortalized by the same research group (Rheinwald Laboratory). This cell line has previously been described to reduce wound contraction and promote confluent epithelial coverage in a mouse model when seeded in a collagen-glycosaminoglycan matrix. ${ }^{24}$ The second gingiva $\mathrm{KC}$ cell line investigated in our study was immortalized with human papillomavirus type 16 (University Medical Center Freiburg) and is referred to in our study as KC-HPV. ${ }^{25}$ The fibroblast cell line used in our study was a human gingiva TERT-immortalized fibroblast cell line (T0026; available from $\mathrm{ABM}$ ) and is further referred to as Fib-TERT.

In this study, organotypic GE models, constructed from either TERT- or HPV-immortalized human gingiva $\mathrm{KC}$ and gingiva fibroblasts, were compared to GE constructed from primary cells with regard to tissue architecture and their ability to secrete (pro-)inflammatory cytokines. Henceforth, the physiological relevance of GE constructed with TERTimmortalized gingiva cell lines was further tested in an in vitro wound healing experiment.

\section{Materials and Methods \\ Cell isolation and culture of primary human gingiva keratinocytes and fibroblasts.}

Healthy human gingiva tissue was used in an anonymous manner in accordance with the "Code for Proper Use of Human Tissues" as formulated by the Dutch Federation of Medical Scientific Organizations (www.fmwv.nl). Primary gingiva $\mathrm{KC}$ and fibroblasts were isolated as previously described for skin. ${ }^{26-28}$ In short, after overnight digestion in dispase II (Sigma-Aldrich) to separate the epithelial sheet from the lamina propria, gingiva $\mathrm{KC}$ (KC-Prim) were isolated from the epithelium with $0.05 \%$ trypsin/EDTA (Gibco) solution. $\mathrm{KC}$ were cultured in $\mathrm{KC}$ medium consisting of DMEM/Ham's F12 (3/1) (Gibco), supplemented with 5\% Fetal Clone III (GE), 1\% penicillin-streptomycin (Gibco), $1 \mu \mathrm{M}$ hydrocortisone (Sigma-Aldrich), $0.1 \mu \mathrm{M}$ insulin (SigmaAldrich), $1 \mu \mathrm{M}$ isoproterenol (Sigma-Aldrich), and $1 \mathrm{ng} / \mathrm{mL}$ epidermal growth factor (EGF) (Sigma-Aldrich). KC-Prim were used until the third passage. Gingival fibroblasts (FibPrim) were isolated from the lamina propria by incubation in a collagenase type II (Gibco) solution and cultured in fibroblast medium consisting of DMEM, supplemented with $5 \%$ Fetal Clone III and 1\% penicillin-streptomycin. The Fib-Prim were used between passage 3 and 4 . 


\section{Culture of immortalized human gingiva keratinocyte and fibroblast cell lines}

Two human gingiva $\mathrm{KC}$ cell lines and one human gingiva fibroblast cell line were used. Cell lines were used for experiments up to passage 50. Human gingiva $\mathrm{KC}$ cell line, OKG4/bmi1/TERT, was immortalized by expression of Telomerase Reverse Transcriptase (purchased from Rheinwald laboratory). ${ }^{29,30}$ In our study, it is referred to as KC-TERT. Human gingiva $\mathrm{KC}$ were immortalized with the human papillomavirus type 16 as described previously (University Medical Center Freiburg) and are referred to in this study as $\mathrm{KC}-\mathrm{HPV}{ }^{25,31,32}$ Both $\mathrm{KC}$ cell lines were cultured in $\mathrm{KC}$ medium as described above. The human gingiva fibroblast cell line was TERT immortalized (T0026, purchased from ABM) and referred to in this study as Fib-TERT. It was cultured in fibroblast medium as described above.

\section{Culture of in vitro organotypic GEs}

GE consisting of a fully differentiated epithelium on a fibroblast-populated collagen hydrogel were constructed as previously described. ${ }^{4}$ Different GE were constructed by combining primary (GE-Prim) $\mathrm{KC}$ and fibroblasts or immortalized $\mathrm{KC}$ and fibroblasts (GE-TERT $=\mathrm{KC}$-TERT + Fib-TERT; GE$\mathrm{HPV}=\mathrm{KC}-\mathrm{HPV}+$ Fib-TERT $)$. In short, a collagen solution was mixed with gingiva fibroblasts $\left(1 \times 10^{5}\right.$ cells $\left./ \mathrm{mL}\right)$ and pipetted into a six-well transwell insert with $0.4 \mu \mathrm{m}$ pores (Corning). The fibroblast-populated hydrogels were cultured overnight submerged in fibroblast medium. $\mathrm{KC}\left(5 \times 10^{5}\right.$ cells/well $)$ were then seeded on top and GE were cultured submerged in $\mathrm{KC}$ medium for 3 days. GE were then lifted to the air-liquid interface and cultured for a further 10 days in differentiation medium consisting of DMEM/Ham's F12 (3/1), supplemented with 1\% Fetal Clone III, $1 \%$ penicillin-streptomycin, $2 \mu \mathrm{M}$ hydrocortisone, $0.1 \mu \mathrm{M}$ insulin, $1 \mu \mathrm{M}$ isoproterenol, $10 \mu \mathrm{M}$ carnitine (SigmaAldrich), $10 \mathrm{mM}$ L-serine (Sigma-Aldrich), $0.4 \mathrm{mmol} \mathrm{L-}$ ascorbic acid (Sigma-Aldrich), and $2 \mathrm{ng} / \mathrm{mL}$ EGF. During this period, the differentiation medium was changed twice.

\section{Cytokine secretion}

Cytokine secretion by fibroblasts: fibroblasts $\left(1 \times 10^{5}\right.$ cells/well) were seeded in a six-well plate (Corning). The next day, the culture medium was refreshed with medium containing 0,100 , or $200 \mathrm{IU} / \mathrm{mL}$ rhTNF- $\alpha$ (Gentaur). Four hours later, the cells were washed with PBS and further cultured for $24 \mathrm{~h}$ in medium without rhTNF- $\alpha$.

Cytokine secretion by $\mathrm{KC}: \mathrm{KC}\left(2.5 \times 10^{5}\right.$ cells/well $)$ were seeded in a six-well plate (Corning). The next day, culture medium was refreshed and $\mathrm{KC}$ were cultured, unstimulated, for a further $24 \mathrm{~h}$.

Cytokine secretion by GE: GE in inserts were transferred to six-well plates containing $1.5 \mathrm{~mL}$ differentiation medium supplemented with 0,100 , or $200 \mathrm{IU} / \mathrm{mL}$ rhTNF- $\alpha$ and further cultured air exposed for $24 \mathrm{~h}$.

All culture supernatants were harvested and stored at $-20^{\circ} \mathrm{C}$ for later use. ${ }^{33}$

\section{Full-thickness wound healing experiment on GES}

Full-thickness wounds (cold injury) were introduced into GE after 10 days culture at the air-liquid interface as described previously. ${ }^{33}$ Cold injury was applied with a metal device (length $2 \mathrm{~cm}$; width $2 \mathrm{~mm}$ ). This device was cooled to $-196^{\circ} \mathrm{C}$ in liquid nitrogen and directly applied with gentle pressure to the GE for $10 \mathrm{~s}$. After wounding, air-exposed culture was continued. Wound closure and soluble mediator secretion were analyzed at day 1,3 , and 7 after wounding. GE were given new culture media $24 \mathrm{~h}$ before collecting the supernatant for soluble mediator analysis at each time interval. For 7-day wounded GE, the culture medium was also refreshed at day 3 after wounding. Culture supernatants were stored at $-20^{\circ} \mathrm{C}$ for analysis with enzyme-linked immunosorbent assay (ELISA).

\section{Metabolic activity assay}

Metabolic activity was analyzed using the MTT assay with biopsies ( $3 \mathrm{~mm}$ diameter) taken from wounded and unwounded parts of the GE and analyzed exactly as previously described in a $2 \mathrm{mg} / \mathrm{mL}$ MTT (Sigma-Aldrich) solution for $1 \mathrm{~h}^{34}$ The biopsies were transferred to an isopropanol/33\% HCL (3:1) solution for $24 \mathrm{~h}$; hereafter, color intensity was measured at $570 \mathrm{~nm}$.

\section{Histology and immunohistochemistry}

GE were fixed in 4\% paraformaldehyde and processed for paraffin embedment. After rehydration, tissue sections $(5 \mu \mathrm{m})$ were stained with hematoxylin and eosin (H\&E) for histological examination or processed for immunohistochemistry (IHC) to study expression of specific proteins. For IHC, after antigen retrieval, sections were incubated for $1 \mathrm{~h}$ with mouse antibodies against human involucrin (Novocastra), K10 (Santa Cruz Biotechnology), K13 (Monosan), vimentin (Dako), or Ki-67 (Dako) as previously described. ${ }^{3,4}$ Collagen type IV (Monosan) and laminin 5 (Dako) IHC were performed on cryosections without antigen retrieval. Hereafter, the sections were washed in PBS and incubated for $30 \mathrm{~min}$ with Envision (Dako), except for K13, which was incubated with PowerVision Poly-HRP (Leica Biosystems) for one hour. Next, the sections were incubated with AEC substrate for 10 minutes followed by hematoxylin staining. The microscopic slides were visualized and recorded with a Nikon Eclipse 80i. Contrast enhancement and quantification of reepithelialization on H\&E sections were done with NISElements software (Nikon Instruments Europe B.V.).

\section{ELISA for cytokine production}

In accordance with the manufacturer's specifications, cytokine CCL2, CXCL8, IL-1 $\alpha$, IL-6, and IL-18 ELISAs were performed using the culture supernatants as previously described. ${ }^{27,35,36}$ The required antibodies and recombinant proteins were supplied by R\&D Systems, Inc., except for CXCL8, which was supplied by Sanquin.

\section{Statistics}

Statistical analysis was performed with the aid of GraphPad Prism, version 6 (GraphPad Software, Inc.). All data represent at least three individual experiments and were analyzed with the Kruskal-Wallis test (keratinocyte cytokine expression) or a two-way ANOVA followed by Sidak's multiple comparison (all other data). Differences were considered significant when $p<0.05$. Data are represented \pm standard error of mean; $*=p<0.05 ; * *=p<0.01 * * *=p<0.001$. 
Results

Comparison of immortalized gingiva cell lines with primary keratinocytes and fibroblasts

To determine how far the immortalized cells represent the morphology of their primary cell counterpart, they were observed with phase contrast light microscopy. Typically, when cultured in a calcium containing medium, KC-Prim proliferate and partially differentiate forming compact colonies (Fig. 1A). ${ }^{37}$ The KC-TERT formed colonies, which were indistinguishable from KC-Prim, whereas $\mathrm{KC}-\mathrm{HPV}$ formed undifferentiated loose colonies with single cells migrating outside of the borders of the colony. The TERTimmortalized gingiva fibroblasts, Fib-TERT, grew as isolated spindle-shaped cells, which were indistinguishable from their primary cell counterpart Fib-Prim (Fig. 2A).

Next, we investigated the ability of the immortalized cells to secrete cytokines. For gingival $\mathrm{KC}$, the basal secretion of proinflammatory cytokines IL- $1 \alpha$ and IL-18 and inflammatory cytokine CXCL8 was determined (Fig. 1B). For all three cytokines, secretion by KC-Prim $>$ KC-TERT $>$ KCHPV, although significant differences were only observed between KC-Prim and KC-HPV. For example, CXCL8 secretion by KC-HPV was 1000-fold lower than KC-Prim.

Since gingival fibroblasts are known to respond to proinflammatory cytokine $\mathrm{TNF}-\alpha$ by secreting inflammatory mediators, ${ }^{4}$ we next investigated the dose-dependent increase in IL-6, CXCL8, and CCL2 secretion in response to TNF- $\alpha$. Fib-TERT showed an identical dose-dependent increase in IL-6 and CXCL8 secretion to that observed with Fib-Prim (Fig. 2B). However, while Fib-TERT showed a dose-dependent increase in CCL2 secretion (trend; $p=0.08$ ), this was not observed with Fib-Prim. No differences were found between the cytokine secretion of Fib-Prim and FibTERT. Taken together, these results indicate that KC-TERT and Fib-TERT very closely resemble their primary cell counterpart when cultured under conventional submerged culture conditions. In contrast, KC-HPV showed significant differences compared to KC-Prim.

\section{GEs constructed with TERT-immortalized cells closely resemble native gingiva}

Native gingiva consists of a differentiated epithelium on a fibroblast-populated (vimentin stained) lamina propria (Fig. 3). The epithelium shows suprabasal expression of the differentiation markers involucrin, K10, and K13. The proliferation marker, $\mathrm{Ki} 67$, is expressed in the basal and first

FIG. 1. Morphology and cytokine secretion of TERTimmortalized gingiva keratinocytes are similar to primary gingiva keratinocytes. Primary and immortalized human gingiva keratinocytes grown under conventional submerged culture conditions. (A) Phase contrast images of primary (KC-Prim), TERT-immortalized (KC-TERT), and HPVimmortalized (KC-HPV) human gingiva keratinocytes. (B) Secretion of IL-1 $\alpha$, IL-18, and CXCL8 by gingiva keratinocytes into culture supernatants over a $24-\mathrm{h}$ culture period. Results are from four individual donors for KC-Prim and six different passages between passage 30 and 50 for the immortalized gingiva keratinocytes KC-TERT and KC-HPV; $* p<0.05 ; * * p<0.01$; Kruskal-Wallis test.
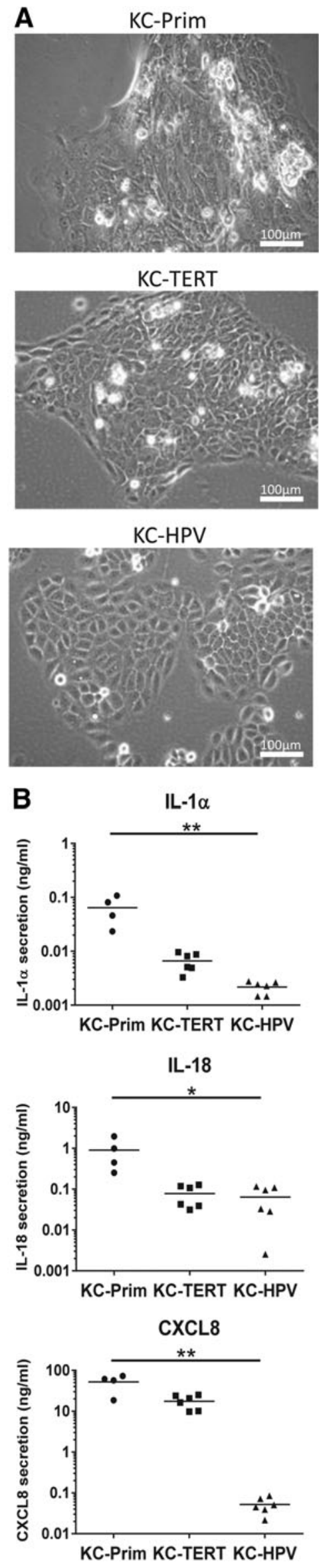

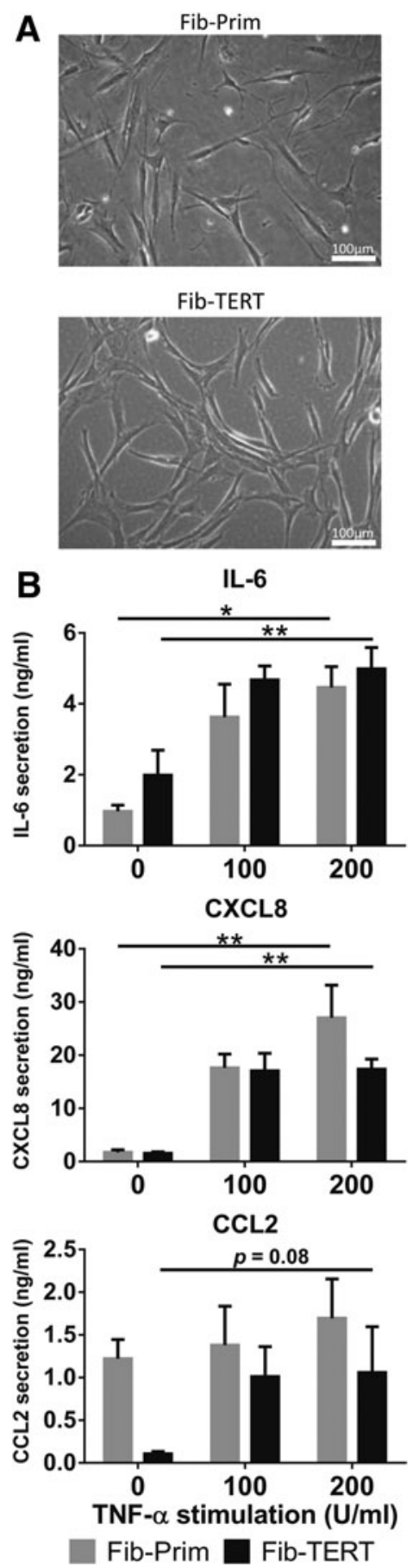

FIG. 2. Morphology and cytokine secretion of TERTimmortalized gingiva fibroblasts are similar to primary gingiva fibroblasts. Primary (Fib-Prim) and TERT-immortalized (Fib-TERT) human gingiva fibroblasts grown under conventional submerged culture conditions. (A) Phase contrast images of Fib-Prim and Fib-TERT. (B) Secretion of IL-6, CXCL8, and CCL2 into culture supernatants by Fib-Prim (gray bars) and Fib-TERT (black bars) exposed to rhTNF- $\alpha$ over $24 \mathrm{~h}$. Data represent the average of at least three individual donors for Fib-Prim and four different passages of FibTERT between passage 20 and $30 \pm$ SEM; $* p<0.05$; $* * p<0.01$; two-way ANOVA for analysis of response to rhTNF- $\alpha$ exposure and Sidak's multiple comparison between Fib-Prim and Fib-TERT. SEM, standard error of mean. suprabasal cell layers. GE were constructed from primary and immortalized cells and compared to native gingiva. GE constructed with primary cells (GE-Prim) as well as TERT keratinocytes and fibroblasts (GE-TERT) very closely resembled native gingiva, although the deep rete ridges were absent in the collagen hydrogel model (Fig. 3). In both GEPrim and GE-TERT, a differentiated stratified epithelium on a fibroblast-populated collagen hydrogel was observed. Involucrin, K10, and K13 expression pattern was similar to that of native gingiva. The proliferation marker, Ki67, was confined to the basal layer. Basement membrane proteins collagen type IV and laminin 5 were expressed at the boundary between the epithelium and the collagen hydrogel layer similar to the native gingiva. In contrast to GE-Prim and GE-TERT, GE constructed with KC-HPV did not form a well-differentiated epithelium. A disorganized multilayer was formed, which lacked typical epithelial differentiation and showed only very low expression of involucrin, K10, and K13 protein and irregular suprabasal expression of proliferation marker Ki67 (Fig. 3A). Interestingly, collagen type IV and laminin 5 were also intermittently expressed within the suprabasal epithelial layers (Fig. 3B). These findings indicate that GE-TERT more closely represents healthy native gingiva and GE-Prim than GE-HPV does, and therefore, only GE-TERT was used in further comparative studies with GE-Prim.

\section{GEs made with TERT-immortalized cells show an inflammatory response and reepithelialize after wounding}

Since GE-TERT very closely resembled the healthy native gingiva architecture, we next investigated whether the construct was able to respond to the proinflammatory cytokine TNF- $\alpha$ and compared this to GE constructed with primary cells. When TNF- $\alpha$ was added to the culture medium of the GE-Prim or GE-TERT, both models responded by increasing secretion of IL-6, CCL2, and CXCL8 in a dose-dependent manner and to the same extent (Fig. 4).

To test the functional aspect of GE-TERT further, fullthickness cold injury wounds (width $3.6 \mathrm{~mm} \pm 1.5 \mathrm{~mm}$ [SD]) were introduced. The histology of the wounded area showed necrotic keratinocytes and fibroblasts (rounded nuclei), while the unwounded region of these same cultures remained similar to that of unwounded GE-TERT (Figs. 3 and 5A). Three days after injury, regenerating epithelium was seen underneath the dead keratinocyte layer on both sides of the wound. One week after injury, the wound had completely reepithelialized (Fig. 5A, B). Fibroblast migration clearly lagged behind reepithelialization with repopulation of the entire collagen hydrogel occurring 7 days after wounding (Fig. 5A). Wound closure in the GE-TERT was also assessed by restoration of metabolic activity (MTT assay). Metabolic activity was drastically decreased in the wounded area directly after wounding and returned to normal after one week, corresponding to the ingrowth of new well-differentiated epithelium and fibroblasts into the wounded area (Fig. 5B).

Since the GE-TERT could respond to proinflammatory cytokine TNF- $\alpha$ by secretion of inflammatory cytokines (Fig. 4), we next investigated whether the GE-TERT could also initiate an inflammatory response after injury. In the 
A
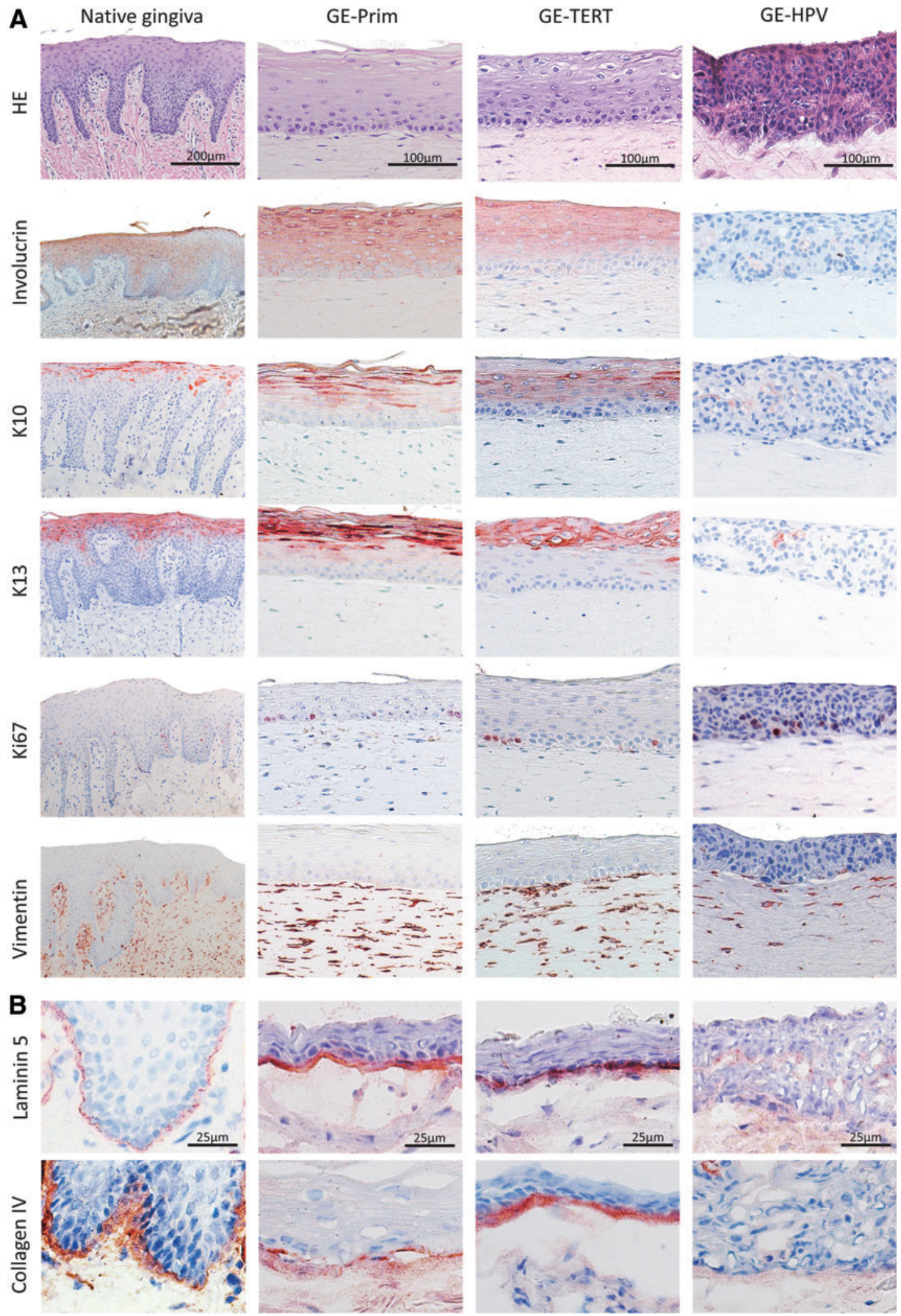

FIG. 3. Full-thickness gingiva equivalents (GE) constructed entirely from cell lines closely represent the native gingiva tissue architecture. GEs were constructed from primary human gingiva keratinocytes and fibroblasts (GE-Prim) or either TERT (GE-TERT)- or HPV (GE-HPV)-immortalized keratinocytes with TERT-immortalized fibroblasts as described in the Materials and Methods section. Figures are representative of at least three independent experiments. (A) Paraffin tissue sections $(5 \mu \mathrm{m})$ were stained with hematoxylin and eosin $(\mathrm{H} \& \mathrm{E})$ to visualize histology or immunohistochemically processed with antibodies directed against epithelial biomarkers (involucrin, K10, or K13), proliferation marker Ki-67, or the fibroblast biomarker vimentin (red immune staining). Scale bar represents $200 \mu \mathrm{m}$ for the native gingiva biopsies and $100 \mu \mathrm{m}$ for the GEs. (B) Immunohistochemistry of basement membrane proteins collagen type IV and laminin 5 on cryosections $(5 \mu \mathrm{m})$. Scale bar represents $25 \mu \mathrm{m}$. Color images available online at www.liebertpub.com/tec 

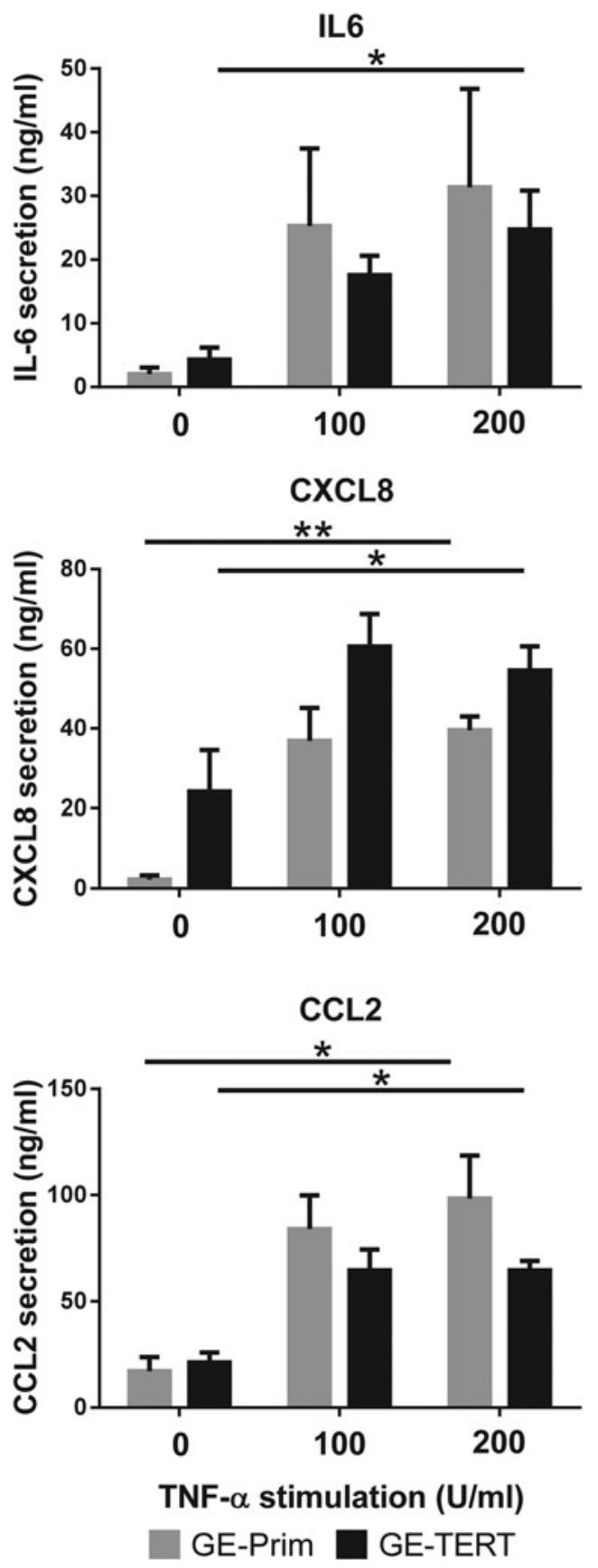

FIG. 4. TERT-immortalized GE secrete inflammatory cytokines in response to rhTNF- $\alpha$ in a similar manner to GE constructed from primary cells. Primary (GE-Prim; gray bars) and TERT-immortalized (GE-TERT, black bars) human GEs were grown as described in the Materials and Methods section and then cultured for $24 \mathrm{~h}$ in the presence or absence of different concentrations of rhTNF- $\alpha$. Cytokine IL-6, CXCL8, and CCL2 secretion into culture supernatants was determined with ELISA. Data represent the average of three individual donors for GE-Prim and three different passages of immortalized cells between passage 30 and $50 \pm$ SEM; $* p<0.05 ; * * p<0.01$; two-way ANOVA for analysis of response to rhTNF- $\alpha$ exposure and Sidak's multiple comparison between GE-Prim and GE-TERT. ELISA, enzyme-linked immunosorbent assay. first day after wounding a large increase in release of cytokines IL-6, CCL20 and CXCL8 were measured. There was less cytokine release during the third day, with only CXCL8 still being significantly increased compared to unwounded GE-TERT. After 1 week, when the wounds had reepithelialized, all cytokine levels had returned to the same basal levels as that of unwounded GE-TERT. CCL2 (Fig. 5) and CCL5 release was not significantly increased after wounding (data not shown). Taken together, these results show that wounding the GE-TERT caused cell death in the affected area and an inflammatory cytokine release. Complete wound healing was observed within 7 days, coinciding with cytokine secretion and metabolic activity returning to prewounded levels.

\section{Discussion}

In this study, we describe a functional full-thickness human GE, which is constructed entirely from immortalized cell lines. The use of TERT-immortalized KC and fibroblasts resulted in GE, which are phenotypically very similar to the native tissue and are able to heal and secrete inflammatory cytokines in response to tissue injury in vitro. GE constructed with KC-HPV showed characteristics representative of squamous cell carcinoma (SCC), indicating that GE-HPV may be suitable as an oral mucosa tumor model.

We have shown that all three cell lines remain stable and functional until at least passage 50. The cells maintain their proliferative potential and notably the KC-TERT keep their ability to differentiate into a stratified epithelium. KC-TERT was able to secrete proinflammatory cytokines and FibTERT was able to respond to proinflammatory TNF- $\alpha$ in a similar manner to Fib-Prim by increasing secretion of inflammatory cytokines. Our results are in line with reports describing cytokine production in primary gingiva $\mathrm{KC}$ and fibroblast cultures. ${ }^{4,38,39}$ Notably, KC-HPV showed reduced cytokine secretion compared to primary $\mathrm{KC}$, which is also in line with reports by others $40-43$

The GE-TERT very closely represented its primary cell counterpart and native gingiva. ${ }^{1,3,5,6}$ When KC-TERT was used for the construction of the organotypic model, it formed a multilayered epithelium with a clear transition from cuboidal to squamous cells. However, the characteristic rete ridges found in native gingiva were absent. This could be due to two reasons: (i) rete ridges are thought to be the result of mechanical forces in vivo, which are absent in the in vitro model $^{44}$ and (ii) the fibroblast populated collagen hydrogel is poured in such a way that a smooth upper surface is created onto which keratinocytes are seeded. In future research, it may be possible to introduce mechanical forces to learn more about the rete ridge development. The stratified squamous epithelium of KC-TERT showed suprabasal expression of differentiation markers involucrin and keratins 10 and 13. Furthermore, the expression of collagen type IV and laminin 5 indicates that keratinocytefibroblast cross talk has occurred to result in deposition of basement membrane proteins. ${ }^{10}$ Clearly, TERT immortalization overcame the cell senescence of primary cells while at the same time maintaining the $\mathrm{KC}$ ability to differentiate and participate in basement membrane formation. When the GE-TERT was stimulated with TNF- $\alpha$ there was a dosedependent increase in the release of inflammatory cytokines 


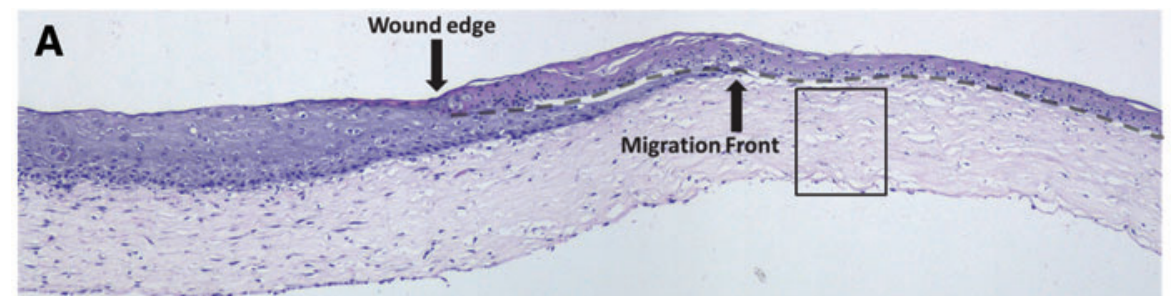

\section{Day 1}
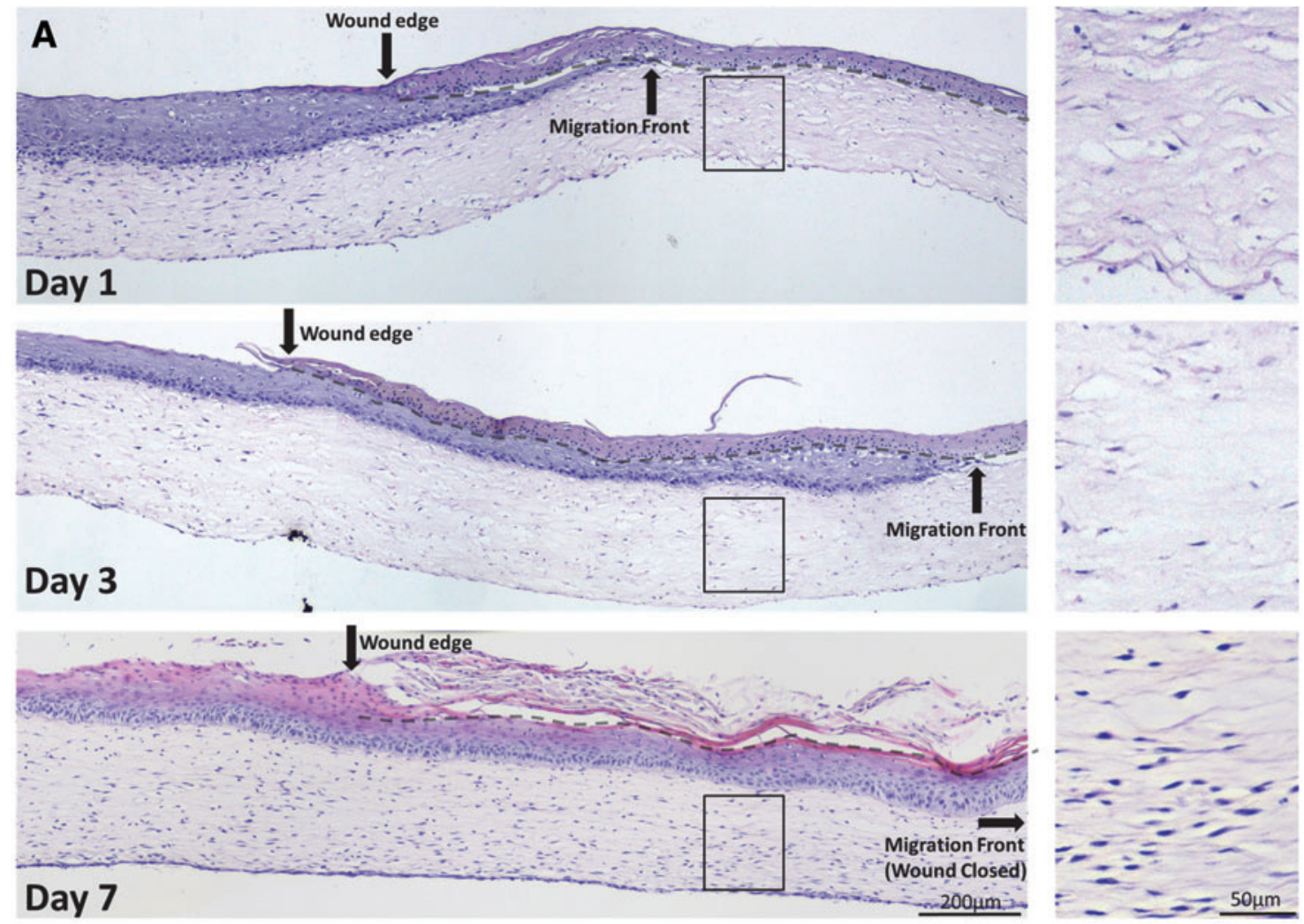

B
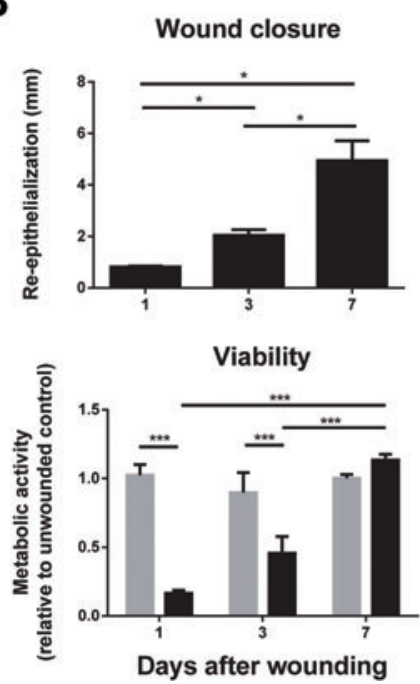

C

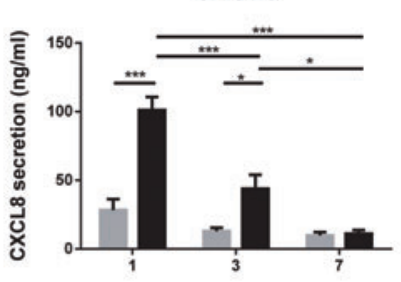

CCL20

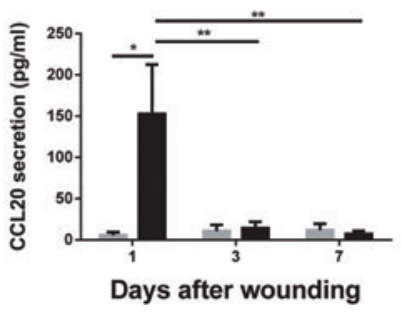

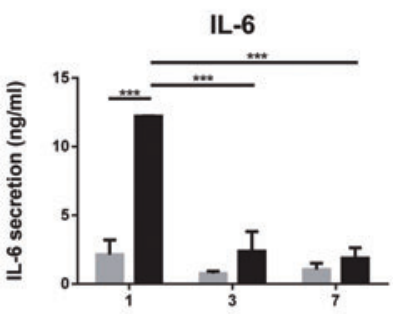

CCL2

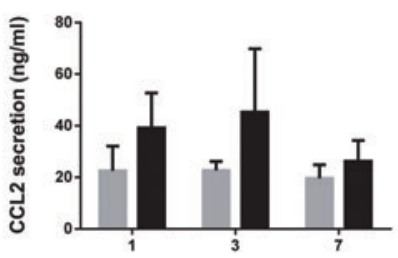

Days after wounding

FIG. 5. TERT-immortalized GE are functional in an in vitro wound-healing assay. Full-thickness freeze wounds were introduced into TERT-immortalized human GE and reepithelialization and fibroblast migration into the wound area as well as inflammatory cytokine secretion were assessed 1,3, and 7 days after introducing the wound. (A) H\&E staining of tissue sections derived from GE-TERT 1, 3, and 7 days after wounding. Black arrow indicates original wound edge; dotted line indicates the interface between the dead epithelial layer and the ingrowing epithelial layer. Inlay shows absence and repopulation of fibroblasts within the original wound in the collagen hydrogel. (B) Upper panel: Quantification of reepithelialization from the wound margins 1, 3, and 7 days after wounding. Lower panel; 3 mm diameter punch biopsies were obtained from the unwounded area (gray bar) and wounded area (black bar) of GE-TERT and analyzed with the MTT mitochondrial activity assay, which represents cell viability. (C) Cytokine secretion by unwounded (gray bars) and wounded (black bars) GE-TERT cultures. Culture supernatants were collected over a 24-h period between 0-1, 2-3, and 67 days after wounding and analyzed by ELISA. All figures represent the average of three independent experiments \pm SEM; $* p<0.05 ; * *<<0.01 ; * * * p<0.001$; two-way ANOVA for response between days and Sidak's multiple comparison between unwounded and wounded areas/cultures. Color images available online at www.liebertpub.com/tec 
IL-6, CXCL8, and CCL2, indicating that the GE-TERT is able to respond to and maintain an immune response. ${ }^{4}$ The release of these cytokines was similar to that of the GEPrim, again illustrating the similarity between primary gingiva cells and the TERT-immortalized cells. Furthermore, we could show that wounding the GE-TERT caused cell death in the affected area, which resulted in inflammatory cytokine release followed by wound closure. The use of GETERT rather than GE-Prim for the wound healing experiment highlights the benefit of using the well-characterized cell lines described in this article. Such large wound healing experiments require many cells making the use of primary gingiva cells not feasible due to the scarcity of gingiva biopsies, their small size, and frequent infected state. Taken together, the GE-TERT is a promising in vitro model to replace GE constructed from primary cells, which is reliant on a constant supply of fresh gingiva tissue. ${ }^{2-7}$

Recently, we described a full-thickness SE constructed from TERT-immortalized human skin cell lines (N/TERT-1 keratinocytes and hTERT BJ-5ta fibroblast), which, similar to the GE-TERT described in this study, also showed excellent physiological similarity to native skin and was able to exert an inflammatory response during wound closure. ${ }^{13}$ Different cytokine release profiles after wounding of oral or skin tissue have been suggested to be involved in the reduced scar formation observed in oral tissue. ${ }^{45}$ Studies comparing the cytokine release of human oral and skin tissue are scarce due to the limited availability of fresh biopsies for such experiments. However, recently we have shown in a comparative study that key cytokines regulating Langerhans cell migration from epithelium to dermis/lamina propria are indeed very different between gingiva and SE derived from primary cells, confirming essential differences in the innate immunology of the two tissues. ${ }^{4}$ The unique phenotype of oral mucosal fibroblasts and $\mathrm{KC}$ underlines the necessity to use tissue-specific cells for such comparative studies. ${ }^{15,46,47}$ In the future, the GE and SE constructed from tissue-specific TERT-immortalized cells will provide valuable physiologically relevant tools to investigate intrinsic differences in wound healing and innate immunology between these two tissues, bypassing the complicated logistics and ethics related to a steady supply of fresh biopsies to the research laboratory. In addition, the GE-TERT cells used in this study would make a logical model for studying in vitro oral implant material interactions since the gingiva is in direct contact with the material in vivo. ${ }^{48}$

In contrast to the GE-TERT, which closely represented the healthy native tissue, the disorganized appearance and the lack of flattened differentiated cells in the epithelium of the GE-HPV more closely represented SCC morphology. HPV is indeed associated with oral SCC in patients, ${ }^{4-51}$ and HPV-immortalized oral $\mathrm{KC}$ have previously been used to investigate SCC. ${ }^{17-20}$ In line with our findings, GE incorporating these HPV-immortalized oral $\mathrm{KC}$ also showed a lack of typical gingival epithelial differentiation. It was shown that the disorganized epithelial morphology found in the GE-HPV is comparable to SCC development. Furthermore, Oda et al. found an invasion of the HPV-immortalized $\mathrm{KC}$ into the collagen hydrogel, which further strengthens the resemblance to SCC. ${ }^{17}$ Indeed we observed depositions of basement membrane proteins collagen type IV and laminin 5 forming within the epithelium of our GE-HPV, and a striking absence of a basement membrane between the epithelium and collagen hydrogel (in contrast to GE-Prim and GE-TERT). Using the same KC-HPV as we used, but in combination with primary gingiva fibroblasts, Roesch-Ely et al. also found reduced K13 expression and reduced squamous differentiation in their GE model. ${ }^{25}$ However, in contrast to our findings, these authors described expression of the differentiation markers K10 and involucrin. Taken together, these findings clearly indicate a reduced ability of the KC-HPV to differentiate and the resemblance of the GEHPV to SCC development. The reduced cytokine expression of the KC-HPV might appear to be counterintuitive for SCC. However, the elevated cytokine expression of SCC in vivo has been suggested to be a result of the tumor-host interaction. ${ }^{42}$ HPV-infected KC actually show reduced expression of inflammatory cytokines. ${ }^{40-43}$ The reduced inflammatory response contributes to the inability of the immune system to clear the HPV infection. ${ }^{52}$ The failure to clear the infection is one factor leading to SCC in vivo. The GE-HPV described in this study is therefore a possible model to investigate SCC development in vitro without the requirement of fresh biopsy material.

In conclusion, we demonstrated that immortalized human gingiva keratinocytes and fibroblasts can be used to make physiologically relevant GE, which resemble either the healthy gingiva or oral SCC development. Since all three of the gingiva cell lines are available for research purposes by the suppliers, it will be possible to transfer the technology to other laboratories that have relevant cell and tissue culture expertise. These organotypic 3D models will provide valuable tools to investigate oral mucosa biology and can also be used as an animal alternative for drug targeting, vaccination studies, microbial biofilm studies, and testing new therapeutics.

\section{Acknowledgments}

The authors would like to thank Chantal van Vliet and Vivian Koopmanschap for assisting with some of the experiments.

\section{Disclosure Statement}

S.G. is cofounder of the VUMC university spin off company A-Skin BV.

\section{References}

1. Presland, R.B., and Dale, B.A. Epithelial structural proteins of the skin and oral cavity: function in health and disease. Crit Rev Oral Biol Med 11, 383, 2000.

2. Dongari-Bagtzoglou, A., and Kashleva, H. Development of a highly reproducible three-dimensional organotypic model of the oral mucosa. Nat Protoc 1, 2012, 2006.

3. Vriens, A.P., et al. Comparison of autologous full-thickness gingiva and skin substitutes for wound healing. Cell Transplant 17, 1199, 2008.

4. Kosten, I.J., Buskermolen, J.K., Spiekstra, S.W., de Gruijl, T.D., and Gibbs, S. Gingiva equivalents secrete negligible amounts of key chemokines involved in langerhans cell migration compared to skin equivalents. J Immunol Res 2015, 627125, 2015.

5. Gibbs, S., and Ponec, M. Intrinsic regulation of differentiation markers in human epidermis, hard palate and buccal mucosa. Arch Oral Biol 45, 149, 2000. 
6. Moharamzadeh, K., Brook, I.M., Van Noort, R., Scutt, A.M., and Thornhill, M.H. Tissue-engineered oral mucosa: a review of the scientific literature. J Dent Res 86, 115, 2007.

7. Moharamzadeh, K., et al. Tissue-engineered oral mucosa. J Dent Res 91, 642, 2012.

8. Shetty, S., and Gokul, S. Keratinization and its disorders. Oman Med J 27, 348, 2012.

9. Moll, R., Divo, M., and Langbein, L. The human keratins: biology and pathology. Histochem Cell Biol 129, 705, 2008.

10. El Ghalbzouri, A., and Ponec, M. Diffusible factors released by fibroblasts support epidermal morphogenesis and deposition of basement membrane components. Wound Repair Regen 12, 359, 2004.

11. Werner, S., Krieg, T., and Smola, H. Keratinocytefibroblast interactions in wound healing. J Invest Dermatol 127, 998, 2007.

12. Pastar, I., et al. Epithelialization in wound healing: a comprehensive review. Adv Wound Care 3, 445, 2014.

13. Reijnders, C.M.A., et al. Development of a full-thickness human skin equivalent in vitro model derived from TERTimmortalized keratinocytes and fibroblasts. Tissue Eng Part A 21, 2448, 2015.

14. Kosten, I.J., Spiekstra, S.W., de Gruijl, T.D., and Gibbs, S. MUTZ-3 derived langerhans cells in human skin equivalents show differential migration and phenotypic plasticity after allergen or irritant exposure. Toxicol Appl Pharmacol 287, 35, 2015.

15. Sriram, G., Bigliardi, P.L., and Bigliardi-Qi, M. Fibroblast heterogeneity and its implications for engineering organotypic skin models in vitro. Eur J Cell Biol 94, 483, 2015.

16. Bao, K., Akguel, B., and Bostanci, N. Establishment and characterization of immortalized gingival epithelial and fibroblastic cell lines for the development of organotypic cultures. Cells Tissues Organs 199, 228, 2014.

17. Oda, D., Bigler, L., Lee, P., and Blanton, R. HPV immortalization of human oral epithelial cells: a model for carcinogenesis. Exp Cell Res 226, 164, 1996.

18. Yoo, G.H., et al. The effects of exogenous p53 overexpression on HPV-immortalized and carcinogen transformed oral keratinocytes. Cancer 94, 159, 2002.

19. Yoo, G.H., et al. Progression of head and neck cancer in an in vitro model. Arch Otolaryngol Head Neck Surg 126, 1313, 2000.

20. Delvenne, P., et al. The organotypic culture of HPVtransformed keratinocytes: an effective in vitro model for the development of new immunotherapeutic approaches for mucosal (pre)neoplastic lesions. Vaccine 19, 2557, 2001.

21. Illeperuma, R.P., et al. Immortalized gingival fibroblasts as a cytotoxicity test model for dental materials. J Mater Sci Mater Med 23, 753, 2012.

22. Millhouse, E., et al. Development of an in vitro periodontal biofilm model for assessing antimicrobial and host modulatory effects of bioactive molecules. BMC Oral Health 14, 80,2014

23. Dickman, C.T.D., Towle, R., Saini, R., and Garnis, C. Molecular characterization of immortalized normal and dysplastic oral cell lines. J Oral Pathol Med 44, 329, 2015.

24. Butler, C.E., Navarro, F.A, Park, C.S., and Orgill, D. P. Regeneration of neomucosa using cell-seeded collagenGAG matrices in athymic mice. Ann Plast Surg 48, 298, 2002.
25. Roesch-Ely, M., et al. Organotypic co-cultures allow for immortalized human gingival keratinocytes to reconstitute a gingival epithelial phenotype in vitro. Differentiation 74, 622, 2006.

26. Waaijman, T., et al. Use of a collagen-elastin matrix as transport carrier system to transfer proliferating epidermal cells to human dermis in vitro. Cell Transplant 19, 1339, 2010.

27. van den Broek, L.J., et al. Differential response of human adipose tissue-derived mesenchymal stem cells, dermal fibroblasts, and keratinocytes to burn wound exudates: potential role of skin-specific chemokine CCL27. Tissue Eng Part A 20, 197, 2014.

28. Kroeze, K.L., et al. Chemokine-mediated migration of skin-derived stem cells: predominant role for CCL5/ RANTES. J Invest Dermatol 129, 1569, 2009.

29. Lindberg, K., and Rheinwald, J.G. Three distinct keratinocyte subtypes identified in human oral epithelium by their patterns of keratin expression in culture and in xenografts. Differentiation 45, 230, 1990.

30. Dickson, M.A., et al. Human keratinocytes that express hTERT and also bypass a p16(INK4a)-enforced mechanism that limits life span become immortal yet retain normal growth and differentiation characteristics. Mol Cell Biol 20, 1436, 2000.

31. Steinberg, T., et al. Analysis of interleukin-1betamodulated mRNA gene transcription in human gingival keratinocytes by epithelia-specific cDNA microarrays. J Periodontal Res 41, 426, 2006.

32. Schulz, S.D., et al. Gene expression analysis of conventional and interactive human gingival cell systems exposed to dental composites. Dent Mater 31, 1321, 2015.

33. Kroeze, K.L., et al. Autocrine regulation of re-epithelialization after wounding by chemokine receptors CCR1, CCR10, CXCR1, CXCR2, and CXCR3. J. Invest Dermatol 132, 216, 2012.

34. Gibbs, S., et al. An epidermal equivalent assay for identification and ranking potency of contact sensitizers. Toxicol Appl Pharmacol 272, 529, 2013.

35. Spiekstra, S.W., et al. Induction of cytokine (interleukin1alpha and tumor necrosis factor-alpha) and chemokine (CCL20, CCL27, and CXCL8) alarm signals after allergen and irritant exposure. Exp Dermatol 14, 109, 2005.

36. van den Broek, L.J., Niessen, F.B., Scheper, R.J., and Gibbs, S. Development, validation and testing of a human tissue engineered hypertrophic scar model. ALTEX 29, $389,2012$.

37. Gibbs, S., Backendorf, C., and Ponec, M. Regulation of keratinocyte proliferation and differentiation by all-transretinoic acid, 9-cis-retinoic acid and 1,25-dihydroxy vitamin D3. Arch Dermatol Res 288, 729, 1996.

38. Cvikl, B., Lussi, A., Moritz, A., Sculean, A., and Gruber, R. Sterile-filtered saliva is a strong inducer of IL-6 and IL-8 in oral fibroblasts. Clin Oral Investig 19, 385, 2014.

39. Hosokawa, I., et al. Proinflammatory effects of muramyldipeptide on human gingival fibroblasts. J Periodontal Res 45, 193, 2010.

40. Woodworth, C.D., and Simpson, S. Comparative lymphokine secretion by cultured normal human cervical keratinocytes, papillomavirus-immortalized, and carcinoma cell lines. Am J Pathol 142, 1544, 1993.

41. De Andrea, M., et al. Alpha- and betapapillomavirus E6/E7 genes differentially modulate pro-inflammatory gene expression. Virus Res 124, 220, 2007. 
42. Smith, C.W., et al. The host environment promotes the development of primary and metastatic squamous cell carcinomas that constitutively express proinflammatory cytokines IL-1alpha, IL-6, GM-CSF, and KC. Clin Exp Metastasis 16, 655, 1998.

43. Amador-Molina, A., Hernández-Valencia, J.F., Lamoyi, E., Contreras-Paredes, A., and Lizano, M. Role of innate immunity against human papillomavirus (HPV) infections and effect of adjuvants in promoting specific immune response. Viruses 5, 2624, 2013.

44. Xiong, X., Wu, T., and He, S. Physical forces make rete ridges in oral mucosa. Med Hypotheses 81, 883, 2013.

45. Chen, L., et al. Positional differences in the wound transcriptome of skin and oral mucosa. BMC Genomics 11, 471, 2010.

46. Turabelidze, A., et al. Intrinsic differences between oral and skin keratinocytes. PLoS One 9, e101480, 2014.

47. Glim, J.E., van Egmond, M., Niessen, F.B., Everts, V., and Beelen, R.H.J. Detrimental dermal wound healing: what can we learn from the oral mucosa? Wound Repair Regen 21, 648, 2013.

48. Torres-Lagares, D., et al. What are the histologic effects of surgical and orthodontic treatment on the gingiva of palatal impacted canines? J Oral Maxillofac Surg 73, 2273, 2015.

49. Kansy, K., Thiele, O., and Freier, K. The role of human papillomavirus in oral squamous cell carcinoma: myth and reality. Oral Maxillofac Surg 18, 165, 2014.
50. Gillison, M.L., Chaturvedi, A.K., Anderson, W.F., and Fakhry, C. Epidemiology of human papillomavirus-positive head and neck squamous cell carcinoma. J Clin Oncol 33, 3235, 2015.

51. Miller, C.S., and Johnstone, B.M. Human papillomavirus as a risk factor for oral squamous cell carcinoma: a metaanalysis, 1982-1997. Oral Surg Oral Med Oral Pathol Oral Radiol Endod 91, 622, 2001.

52. Moody, C.A. and Laimins, L.A. Human papillomavirus oncoproteins: pathways to transformation. Nat Rev Cancer 10, 550, 2010.

Address correspondence to: Susan Gibbs, PhD Department of Oral Cell Biology Academic Centre for Dentistry Amsterdam (ACTA)

Room 11N69

Gustav Mahlerlaan 3004 Amsterdam 1081 LA

The Netherlands

E-mail: s.gibbs@acta.nl

Received: February 17, 2016

Accepted: June 20, 2016

Online Publication Date: August 2, 2016 